Geometric Continuous Patch Complexes

by

Jörg M. Hahn 
z162491x 


\section{Geometric Continuous Patch Complexes}

by

Jörg M. Hahn

Department of Mathematics and Statistics

Brunel University

Uxbridge UB8 3PH, England

This work was supported by the Science and Engineering Research Council grant GR/D/77148.

The author is greatly indebted to Dr. J. A. Gregory for the arrangement of this project and his invaluable advice.

Presented at Oberwolfach 10 February 1987.

Submitted for Publication in Computer Aided Geometric Design. 



\title{
Geometric Continuous Patch Complexes
}

\author{
By
}

Jörg M. Hahn.

\begin{abstract}
$\underline{\text { Abstract }}$
A theory of geometric continuity of arbitrary order is presented. Conditions of geometric continuity at a vertex where a number of patches meet are investigated. Geometric continuous patch complexes are introduced as the appropriate setting for the representation of surfaces in CAGD. The theory is applied to the modelling of closed surfaces and the fitting of triangular patches into a geometric continuous patch complex.
\end{abstract}

$\underline{\text { Key words }}$

Computer Aided Geometric Design

Geometric Continuity 



\section{1. $\underline{\text { Introduction }}$}

At the conference on surfaces in computer aided geometric design (CAGD) in Oberwolfach 1984, [Barnhill '85] presented eight open research questions. The last three of them are:

- geometric continuity

- modelling closed surfaces,

- composing rectangular and triangular patches.

The keystone of these problems is geometric continuity. The other two problems are the challenge for any theory of geometric continuity: the theory must prove its usefulness in resolving them.

The basic concepts of geometric continuity, geometric characterisations of first and second order continuity and the reparameterization approach for continuity of arbitrary order, were already introduced by [Vernon et al. '76]. These ideas have been further developed by [Barsky and DeRose '85], [Höllig '86], and others. [DeRose '85] has attempted to build a theory of geometric continuity on manifold theoretic terms.

In fact, differential topology provides the proper means to deal with geometric continuity. This must be adapted to the needs of CAGD.

This article presents a theory of geometric continuity of arbitrary order that is capable of resolving the two practical problems above.

Section 2 starts with the definition of parametric surface patches suitable for CAGD. In section 3, geometric continuity of adjoining patches is introduced, based on the existence of a reparameterization. Also more practical characterizations are given. Section 4 defines geometric continuity at a corner. Thereupon, in section 5, geometric continuous patch complexes are introduced as the appropriate setting for the representation of surfaces in CAGD. 
Section 6 provides the instruments to investigate, in section 7 , conditions of geometric continuity at a corner.

Sections 8 and 9 outline the application of the theory to the modelling of closed surfaces and the fitting of triangular patches into a geometric continuous patch complex.

Finally, an equivalent characterization of geometric continuity in terms of geometric invariants is described in an appendix.

\section{Surface Patches for CAGD}

2.1 Definition; A domain is a closed subset. $\Delta$ of $\mathbb{R}^{2}$, bounded by a number of edges $E_{i}$ that are regularly $C^{k}$-parameterized as $E_{i}(s), s \in[0,1]$.

$\mathrm{A} \underline{\mathrm{C}}^{\underline{\mathrm{k}}}$-patch is a map $\mathrm{p}: \Delta \rightarrow \mathbb{R}^{3}$ that is k-times continuously differentiable on $\Delta$ and whose differential $\partial \mathrm{p}$ has rank 2 for all points of $\Delta$.

The tangent sector of $\mathrm{p}$ at a boundary point $\mathrm{C}$ of $\Delta$ is the set of all tangent vectors $(\mathrm{p} \text { o } \mathrm{c})^{\prime}{ }_{(0)}$ of curves $\mathrm{c}:[0,1] \rightarrow \Delta$ starting at $\mathrm{C}=\mathrm{c}(0)$.

2.2 Remarks: The rank condition excludes cones, cusp ridges and other kinds of singularities. It guarantees that the tangent plane $\partial \mathrm{p}_{\mid \mathrm{X}}\left(\mathbb{R}^{2}\right)$ is well-defined for all $\mathrm{X} \in \Delta$ and that the tangent sector at a convex corner is convex.

The definition allows for self-intersections, mainly because there is no practicable criterion to exclude them, and because they can occur in some applications. This causes no problems in theoretical considerations, if an intersection point is treated separately according to the different leaves it belongs to, i.e. if the intersecting leaves are considered being disjoint. That is the reason why the tangent plane was attributed to. the point of the domain rather than to its image on the surface.

\section{Geometric Continuity of Adjoining Patches}

In practice it is impossible to describe a complicated surface by a single patch. Instead, the surface will be composed of several patches. This imposes the 
the question of how to glue them together.

The only reasonable assumption is that, locally, a point of the composed surface cannot be distinguished from a point of a patch. The characteristic shared by all points of patches is that they admit locally a parameterization.

Thus, geometric continuity is, in essence, the existence of a (local) $\underline{\text { reparameterization.. }}$

This is also the principle that underlies the work of [Vernon et al. '76 ] and others. The exposition here builds on [Gregory and Hahn '86], particularly in employing the machinery of (total) derivatives.

3.1 Definition Let $\Delta_{1}$ and $\Delta_{2}$ be domains with edges $\mathrm{E}_{1}(\mathrm{~s})$ and $\mathrm{E}_{2}(\mathrm{~s})$ respectively.

$A \underline{C}^{\underline{k}}$-connecting diffeomorphism from $E_{1}$ to $E_{2}$ is a $C^{k}$-diffeomorphism $\phi$ defined in a neighbourhood of $E_{1}$ such that $\phi\left(E_{1}(s)\right){ }^{=} E_{2}(s)$ and $\phi$ maps interior points of $\Delta_{1}$ into the exterior of $\Delta_{2}$.

Two $\mathrm{C}^{\mathrm{k}}$-patches $\mathrm{p}_{1}: \Delta_{1} \rightarrow \mathbb{R}^{3}$ and $\mathrm{p}_{2}: \Delta_{2} \rightarrow \mathbb{R}^{3}$ join with geometric continuity $\left(\mathrm{GC}^{\underline{\mathrm{k}}}\right)$ along edges $E_{1}, E_{2}$, if there exists a $C^{k}$-connecting diffeomorphism $\phi$ from $E_{1}$ to $\mathrm{E}_{2}$ such that the derivatives up to order $\mathrm{k}$ of patch pi and the composed map $\mathrm{p}_{2}$ o $\phi$ coincide along edge $\mathrm{E}_{1}$ :

$$
\partial^{j} p_{1 \mid E_{1}(s)}=\partial^{j}\left(p_{2} \circ \phi\right) \mid E_{1}(s), \text { for } j=0, \ldots, k \text { and } s \in[0,1] .
$$

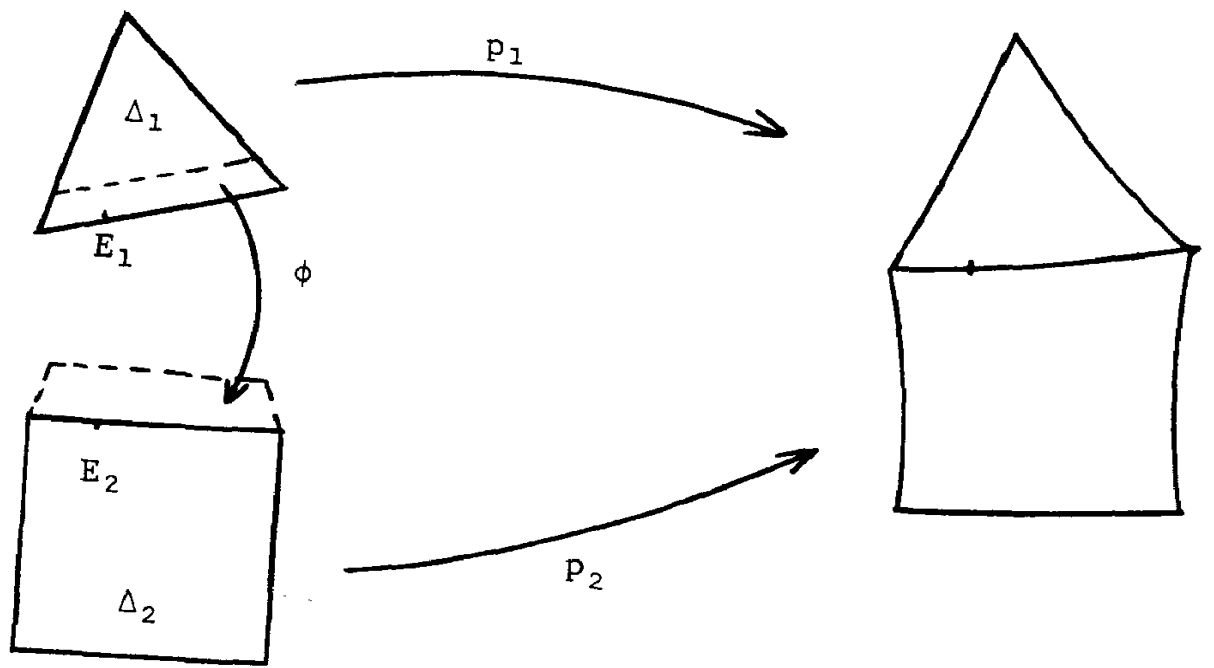

Fig. 1 
With this definition, the map defined in a neighbourhood of edge $\mathrm{E}$ by

$$
\mathrm{X} \rightarrow\left\{\begin{array}{l}
\mathrm{p}_{1}(\mathrm{x}), \text { for } \mathrm{x} \quad \Delta_{1} \\
\mathrm{p}_{2} \circ \phi(\mathrm{x}), \text { for } \mathrm{x} \notin \Delta_{1}, \phi(\mathrm{x}) \in \Delta_{2}
\end{array}\right.
$$

is a $C^{k}$-parameterization of the union of (the ranges of) the patches $p_{1}, p_{2}$ near the common boundary curve $\mathrm{p}_{1} \circ \mathrm{E}_{1}(\mathrm{~s})=\mathrm{p}_{2} \circ \mathrm{E}_{2}(\mathrm{~s})$, see fig. 1 .

It would be somewhat awkward if always the entire connecting diffeomorphism needs to be known. Evaluating $\partial^{\mathrm{j}}\left(\mathrm{p}_{2} \circ \phi\right)$ via the chain rule shows that only its derivatives along the edge are required, and these can be provided much easier.

\subsection{Lemma:}

Let $E_{1}(S)$ and $E_{2}(s)$ be edges of domains $\Delta_{1}, \Delta_{2}$ respectively. Furthermore let $\mathrm{U}(\mathrm{s})$ be a $\mathrm{C}^{\mathrm{k}-1}$-vector field along $\mathrm{E}_{1}(\mathrm{~s})$, transversal and inward pointing, and let $\mathrm{V}^{(1)}(\mathrm{s}), . ., \mathrm{V}^{(\mathrm{k})}$ (s) be vector fields along $\mathrm{E}_{2}(\mathrm{~s})$ such that $\mathrm{V}^{(\mathrm{j})}$ (s) is $\mathrm{C}^{\mathrm{k}-\mathrm{j}}$ and $V^{(1)}$ (s) is transversal to $E_{2}(s)$ and outward pointing.

Then there exists a $C^{k}$-connecting diffeomorphism $\phi$ from $E_{1}$ to $E_{2}$ with

$$
\left.\partial^{\mathrm{j}_{\phi}}\right|_{\mathrm{E}_{1}(\mathrm{~s})}(\mathrm{U}(\mathrm{s}), \ldots, \quad \mathrm{U}(\mathrm{s})) \quad=\mathrm{v}^{(\mathrm{j})}(\mathrm{s}) \quad \text { for } \quad \mathrm{j}=1, \ldots, \mathrm{k} .
$$

Proof: If all vector fields are $\mathrm{C}^{\mathrm{k}}$, then a diffeomorphism can be defined simply by

$$
\phi\left(\mathrm{E}_{1}(\mathrm{~s})+\mathrm{tU}(\mathrm{s})\right):=\mathrm{E}_{2}(\mathrm{~s})+\sum_{\mathrm{j}=1}^{\mathrm{k}} \frac{\mathrm{t}^{\mathrm{j}}}{\mathrm{j} !} \mathrm{v}(\mathrm{j}) \text { (s) . }
$$

To construct a diffeomorphism under the weaker continuity conditions, define integral operators for univariate functions $\mathrm{f}$ :

$$
\begin{aligned}
& \mathrm{I}[\mathrm{f}](\mathrm{s}, \mathrm{t}):=\int_{\mathrm{s}}^{\mathrm{s}+\mathrm{t}} \mathrm{f}, \\
& \mathrm{I}^{(\ell)}[\mathrm{f}](\mathrm{s}, \mathrm{t}):=\int_{0}^{\mathrm{t}} \mathrm{I}^{(\ell-1)}[\mathrm{f}](\mathrm{s}, \cdot), \text { for } \quad \ell=2, . ., \mathrm{k}
\end{aligned}
$$

These satisfy $\frac{\partial^{\mathrm{j}}}{\partial \mathrm{t}^{\mathrm{j}}} \mathrm{I}^{(\ell)}[\mathrm{f}](\mathrm{s}, 0)=0$, for $\mathrm{j}<\ell, \quad$ and $\quad \frac{\partial^{\ell}}{\partial \mathrm{t}^{\ell}} \mathrm{I}^{(\ell)}[\mathrm{f}](\mathrm{s}, 0)=\mathrm{f}(\mathrm{s})$.

Define $C^{\mathrm{k}}$-maps $\phi_{2}^{(\ell)}$ recursively by

$$
\begin{aligned}
& \phi_{2}^{(1)}(\mathrm{s}, \mathrm{t}):=\mathrm{E}_{2}(\mathrm{~s})+\mathrm{I}\left[\mathrm{v}^{(1)}\right](\mathrm{s}, \mathrm{t}), \\
& \phi_{2}^{(1)}(\mathrm{s}, \mathrm{t}):=\phi_{2}^{(\ell-1)}(\mathrm{s}, \mathrm{t})+\mathrm{I}^{(\ell)}\left[\mathrm{v}^{(\ell)}-\frac{\partial^{\ell}}{\partial \mathrm{t}^{\ell}} \phi_{2}^{(\ell-1)}(., 0)\right](\mathrm{s}, \mathrm{t}) \\
& (\mathrm{k}) \quad \text { for } \quad \ell=2 \mathrm{k}
\end{aligned}
$$


By induction one shows that

$$
\frac{\partial^{\mathrm{j}}}{\partial \mathrm{t}} \phi_{2}(\mathrm{~s}, 0)=\mathrm{v}^{(\mathrm{j})}(\mathrm{s}) \text { for } \mathrm{j} \leq \mathrm{k} \text {. }
$$

Similarly there is a $C^{\mathrm{k}}$-map $\phi_{1}$ such that $\phi_{1}(\mathrm{~s}, 0)=\mathrm{E}_{1}(\mathrm{~s}), \frac{\partial}{\partial \mathrm{t}} \phi_{1}(\mathrm{~s}, 0)=\mathrm{U}(\mathrm{s})$ and $\frac{\partial^{\mathrm{j}}}{\partial \mathrm{t}^{\mathrm{j}}} \phi_{1}(\mathrm{~s}, 0)=0$ for $2 \leq \mathrm{j} \leq \mathrm{k}$.

Then $\phi:=\phi_{1} \circ \phi_{1}^{-1}$ is a $\mathrm{C}^{\mathrm{k}}$-diffeomorphism in a neighbourhood of edge $\mathrm{E}_{1}$ and has the prescribed derivatives.

Combining lemma 3.2 with the chain rule and observing that only derivatives in a transversal direction need to be known (differentiate along the edge), gives the following handy characterization of $\mathrm{GC}^{\mathrm{k}}$, here only written for $\mathrm{k}=1,2$.

3.3 Corollary: Two patches $\mathrm{p}_{1}, \mathrm{p}_{2}$ join $\mathrm{GC}^{1}$ along edges $\mathrm{E}_{1}(\mathrm{~s}), \mathrm{E}_{2}(\mathrm{~s})$ iff there exists a $\mathrm{C}^{0}$-vector field $\mathrm{U}(\mathrm{s})$, transversal along $\mathrm{E}_{1}(\mathrm{~S})$ and inward pointing, and a $\mathrm{C}^{0}$-vector field $\mathrm{V}(\mathrm{s})$, transversal along $\mathrm{E}_{2}(\mathrm{~s})$ and outward pointing, such that

$$
\partial \mathrm{p}_{1 \mid \mathrm{E}_{1}(\mathrm{~s})} \mathrm{U}(\mathrm{s})=\partial \mathrm{p}_{2 \mid \mathrm{E}_{2}(\mathrm{~s})} \mathrm{V}(\mathrm{s}) .
$$

The patches join $\mathrm{GC}^{2}$ iff these vector fields are $\mathrm{C}^{1}$ and in addition there exists a $\mathrm{C}^{\circ}$-vector field $\mathrm{W}(\mathrm{s})$ such that

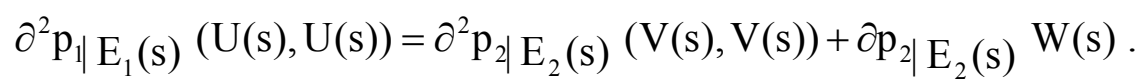

The $\mathrm{GC}^{1}$-condition accords with that of [Herron '85]. The $\mathrm{GC}^{2}$-condition is the starting point for the construction of $\mathrm{C}^{2}$-polygonal patches in [Gregory and Hahn '87] of these proceedings.

\subsection{Example:}

Let $\mathrm{p}_{1}, \mathrm{p}_{2}$ be patches on the unit square or the triangle with vertices $(0,0)$, $(1,0),(0,1)$.

Then with $\mathrm{U}(\mathrm{s})=(1,0), \mathrm{V}(\mathrm{s})=\left(\mathrm{v}_{1}(\mathrm{~s}), \mathrm{v}_{2}(\mathrm{~s})\right), \mathrm{W}(\mathrm{s})=\left(\mathrm{w}_{1}(\mathrm{~s}), \mathrm{w}_{2}(\mathrm{~s})\right)$, the conditions for $\mathrm{GC}^{2}$ along edges $(0, \mathrm{~s}),(\mathrm{s}, 0)(\mathrm{s} €[0,1])$ are $\left(\partial_{1,0}\right.$ etc stands for partial differentiation): 


$$
\begin{aligned}
& \mathrm{v}_{2}(\mathrm{~s})<0 \\
& \mathrm{p}_{1}(0, \mathrm{~s})=\mathrm{p}_{2}(\mathrm{~s}, 0) \\
& \partial_{1,0} \mathrm{p}_{1}(0, \mathrm{~s})=\mathrm{v}_{1}(\mathrm{~s}) \partial_{1,0} \mathrm{p}_{2}(\mathrm{~s}, 0)+\mathrm{v}_{2}(\mathrm{~s}) \partial_{0,1} \mathrm{p}_{2}(\mathrm{~s}, 0) \\
& \partial_{2,0} \mathrm{p}_{1}(0, \mathrm{~s})=\mathrm{v}_{1}(\mathrm{~s})^{2} \partial_{2,0} \mathrm{p}_{2}(\mathrm{~s}, 0)+2 \mathrm{v}_{1}(\mathrm{~s}) \mathrm{v}_{2}(\mathrm{~s}) \partial_{1,1} \mathrm{p}_{2}(\mathrm{~s}, 0) \\
& \quad+\mathrm{v}_{2}^{2}(\mathrm{~s}) \partial_{0,2} \mathrm{p}_{2}(\mathrm{~s}, 0) \\
& \quad+\mathrm{w}_{1}(\mathrm{~s}) \partial_{1,0} \mathrm{p}_{2}(\mathrm{~s}, 0)+\mathrm{w}_{2}(\mathrm{~s}) \partial_{0,1} \mathrm{p}_{2}(\mathrm{~s}, 0)
\end{aligned}
$$

These conditions with 'shape parameters' $\mathrm{v}_{1}, \mathrm{v}_{2}, \mathrm{w}_{1}, \mathrm{w}_{2}$ are similar to those derived by [H811ig '86], following [DeRose '85].

In view of lemma 3.2, the connecting diffeomorphisms could be suppressed in the following considerations, replacing them by a set of vector fields. They are used, however, mainly because they provide a more concise notation.

\section{Geometric Continuity at a Vertex}

Now consider a number of $\mathrm{C}^{\mathrm{k}}$-patches $\mathrm{p}_{\mathrm{i}}: \Delta_{\mathrm{i}} \cdot \rightarrow \mathbb{R}^{3}, \mathrm{i}=1, . ., \mathrm{n}$, meeting at a common vertex $Q \in \mathbb{R}^{3}$ and such that subsequent patches join with $\mathrm{GC}^{\mathrm{k}}$, i.e. there are corner points $C_{i}$ of adjacent edges $E_{i+}, E_{. i+}, E_{i-}$ of $\Delta_{i}$. such that $p_{i}\left(C_{i}\right)=Q, i=1, \ldots, n$

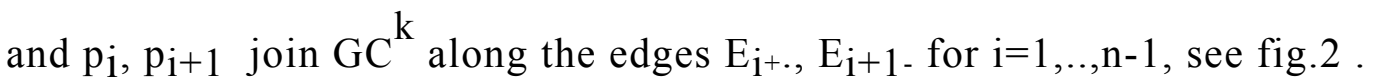

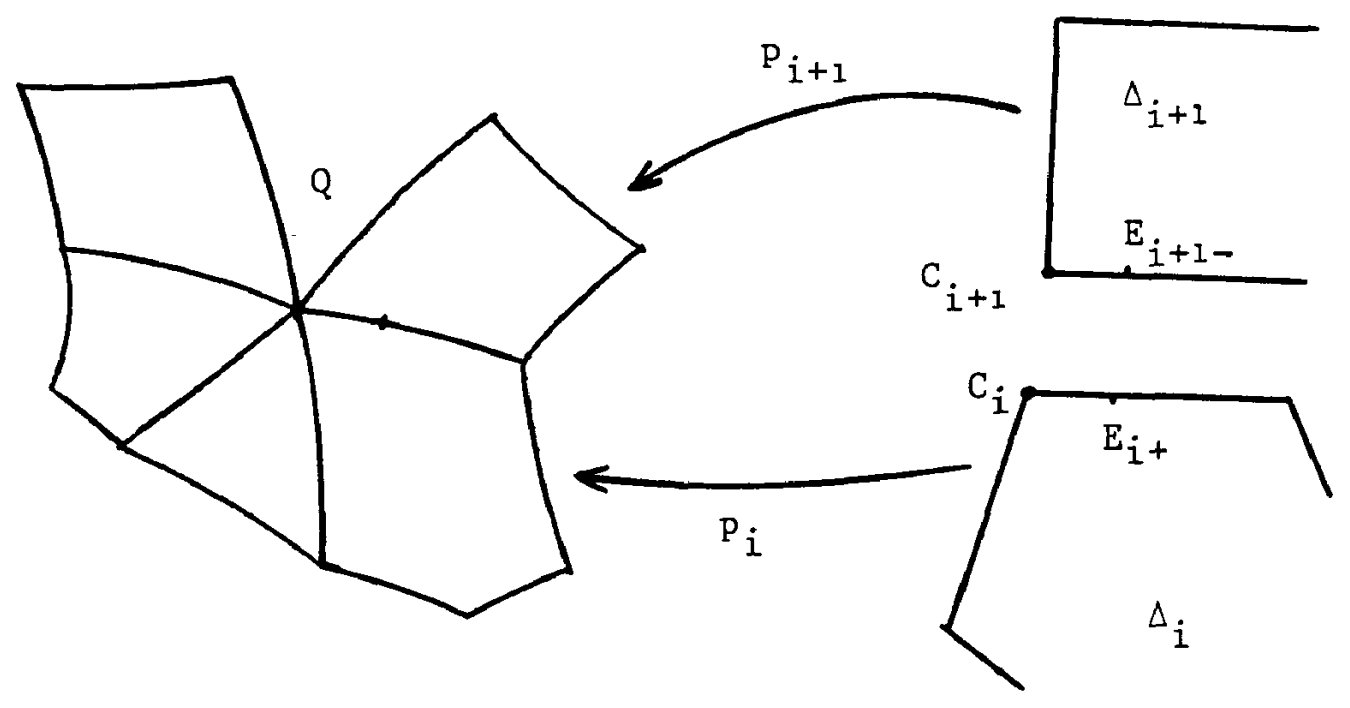

Fig. 2 
4.1 Definition: The patches $\mathrm{p}_{1}, \ldots, \mathrm{p}_{\mathrm{n}}$ meet with geometric continuity $\left(\mathrm{GC}^{\underline{\mathrm{k}}}\right)$ with a (convex/non-convex) corner at $\mathrm{Q}$, if in addition the union of the tangent sectors of $p_{i}$ at $C_{i}(i=1, \ldots, n)$ is a proper subset of the tangent plane at Q (convex or non-convex).

The patches $\mathrm{p}_{1}, . ., \mathrm{p}_{\mathrm{n}}$ surround the vertex $\mathrm{Q}$ with geometric continuity $\left(\mathrm{GC}^{\underline{\mathrm{k}}}\right)$, if also $\mathrm{p}_{\mathrm{n}}, \mathrm{p}_{1}$ join $\mathrm{GC}^{\mathrm{k}}$ along edges $\mathrm{E}_{\mathrm{n}+}, \mathrm{E}_{1}$ - and the tangent sectors of the patches do not overlap.

The patches $\mathrm{p}_{1}, \ldots, \mathrm{p}_{\mathrm{n}}$ join with geometric continuity $\left(\mathrm{GC}^{\mathrm{k}}\right)$ at the vertex $\mathrm{Q}$, if either they meet $\mathrm{GC}^{\mathrm{k}}$ with a corner at $\mathrm{Q}$ or they surround $\mathrm{Q}$ with $\mathrm{GC}^{\mathrm{k}}$.

4.2 Remark: If patches $\mathrm{p}_{1}, \ldots, \mathrm{p}_{\mathrm{n}}$ join $\mathrm{GC}^{\mathrm{k}}$ at a vertex $\mathrm{Q}$, then their union admits a $\mathrm{C}^{\mathrm{k}}$-parameterization around the vertex, e.g. the inverse of the orthogonal projection to the tangent plane at Q.

\section{Geometric Continuous Patch Complexes}

Now the definition of a surface appropriate for CAGD can be given.

5.1 Definition: Let $\mathrm{p}_{\mathrm{i}}: \Delta_{\mathrm{i}} \rightarrow \mathbb{R}^{3}, \mathrm{i}=1, . ., \mathrm{N}$ be $\mathrm{C}^{\mathrm{k}}$-patches and let $\mathrm{E}_{\mathrm{ij} . . .}, \mathrm{j}=1, . ., \mathrm{N}$ denote the edges of $\Delta_{\mathrm{i}}$.

A connecting relation is a relation $\sim$ between the edges that is symmetric, nonreflexive and such that an edge is related to at most one other edge.

A geometric continuous $\left(\mathrm{GC}^{\mathrm{k}}\right)$ patch complex consists of patches $\mathrm{p}_{1}, \ldots, \mathrm{p}_{\mathrm{N}}$ and a connecting relation $\sim$ such that

(i) $\mathrm{p}_{\mathrm{i}_{1}}, \mathrm{p}_{\mathrm{i}_{2}}$ join $\mathrm{GC} \mathrm{k}^{\mathrm{k}}$ along $\mathrm{E}_{\mathrm{i}_{1}, \mathrm{j} 1}, \mathrm{E}_{\mathrm{i}_{2}, \mathrm{j}_{2}}$ whenever $\mathrm{E}_{\mathrm{i}_{1}, \mathrm{j}_{1}} \sim \mathrm{E}_{\mathrm{i}_{2}, \mathrm{j}_{2}}$,

(ii) if a number of patches subsequently join $\mathrm{GC}^{\mathrm{k}}$ with a common vertex, then they join $\mathrm{GC}^{\mathrm{k}}$ at this vertex.

5.2 Remarks: Every point in the union of the patches of a $\mathrm{GC}^{\mathrm{k}}$-patch complex admits locally a $\mathrm{C}^{\mathrm{k}}$-parameterization, i.e. the union of these patches is an 'immersed $\mathrm{C}^{\mathrm{k}}$-surface with piecewise $\mathrm{C}^{\mathrm{k}}$-boundary ${ }^{1}$ in the sense of differential topology, of e.g. [Hirsch '76]. 
The term 'complex' indicates that a patch complex consists of dissimilar parts, namely patches and connecting relations. An assembly of patches can give rise to different patch complexes, depending on the connecting relations imposed.

6. $\quad \underline{\text { Jets }}$

6.1 Definition: Let $\mathrm{f}$ be a $\mathrm{C}^{\mathrm{k}}$-function and $\mathrm{X}$ a point of its domain. The $\mathrm{k}$-jet of $\mathrm{f}$ at $\mathrm{X}$ is the set of all derivatives up to order $\mathrm{k}$ at $\mathrm{X}$ :

$$
\mathrm{j}^{\mathrm{k}} \mathrm{f}_{\mid \mathrm{X}}:=\left(\partial^{\mathrm{j}} \mathrm{f}_{\mid X}\right) \mathrm{j}=0, . ., \mathrm{k} \text {. }
$$

Jets have been used in differential topology with a slightly more abstract definition, of [Hirsch '76]. They are like a high level programming language adapted to deal with geometric continuity, instead of the intermediate level language of (total) derivatives or the machine language of partial derivatives.

6.2 Notes: Many useful properties of jets are directly inherited from properties of the underlying functions:

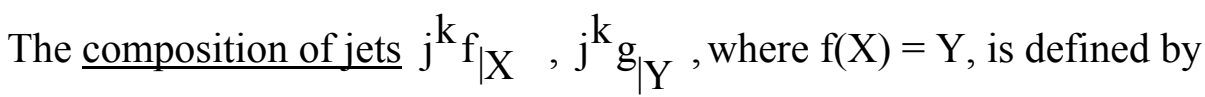

$$
\mathrm{j}^{\mathrm{k}} \mathrm{g}_{\mid \mathrm{Y}} \circ \mathrm{j}^{\mathrm{k}} \mathrm{f}_{\mid \mathrm{X}}:=\mathrm{j}^{\mathrm{k}}(\mathrm{g} \circ \mathrm{f})_{\mid X}
$$

This is well-defined since all derivatives occuring within the right-hand side of this formula can be computed by repeated application of the chain rule, involving only derivatives up to order $\mathrm{k}$ of $\mathrm{f}$ and $\mathrm{g}$, which are given by their k-jets. For instance, the $\mathrm{GC}^{\mathrm{k}}$-condition (def. 3.1) can now be written as

$$
\mathrm{j}^{\mathrm{k}} \mathrm{p}_{1 \mid \mathrm{E}(\mathrm{s})}=\mathrm{j}^{\mathrm{k}} \mathrm{p}_{2 \mid \mathrm{E}_{2}(\mathrm{~s})}{ }^{\circ} \mathrm{j}^{\mathrm{k}} \phi_{\left.\right|_{\mathrm{E}_{1}(\mathrm{~s})}} .
$$

The composition of jets is associative.

The neutral element with respect to jet composition is the jet of the identity map at the point in consideration;

$$
\mathrm{j}^{\mathrm{k}} \mathrm{id}_{\mid Y} \circ \mathrm{j}^{\mathrm{k}} \mathrm{f}_{\mid X}=\mathrm{j}^{\mathrm{k}} \mathrm{f}_{\mid X} \circ \mathrm{j} \mathrm{kdd}_{\mid X}=\mathrm{j}^{\mathrm{k}} \mathrm{f}_{\mid X}
$$

A jet $j^{k} f_{\mid X}$ is left-invertible, if there exists a jet $j^{k} g_{\mid Y}$ such that

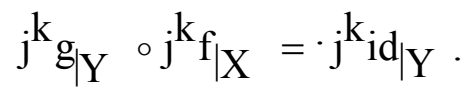

Similarly, $\mathrm{j}^{\mathrm{k}_{\mid \mathrm{Y}}}$ is right-invertible.

A jet is invertible, if it is left- and right-invertible. 
Note that a jet $\mathrm{j}^{\mathrm{k}} \mathrm{f}_{\mid \mathrm{X}}$ is left-invertible (right-invertible) if the differential $\partial \mathrm{f}_{\mid \mathrm{X}}$ is one-to-one (onto).

\section{Conditions of Geometric Continuity at a Vertex}

Let $\mathrm{p}_{1}, ., \mathrm{p}_{\mathrm{n}}$ be patches surrounding a vertex $\mathrm{Q}=\mathrm{p}_{\mathrm{i}}\left(\mathrm{C}_{\mathrm{i}}\right)$ with $\mathrm{GC}^{\mathrm{k}}$. The $\mathrm{GC}^{\mathrm{k}}$-conditions between subsequent patches are, at the vertex:

$$
\begin{aligned}
& \mathrm{j}^{\mathrm{k}} \mathrm{P}_{1 \mid \mathrm{C}_{1}}=\mathrm{j}^{\mathrm{k}} \mathrm{P}_{2 \mid \mathrm{C}_{2}} \circ \mathrm{j}^{\mathrm{k}} \phi_{2,1 \mid \mathrm{C}_{1}}, \\
& \mathrm{j}^{\mathrm{k}} \mathrm{p}_{\mathrm{n}-1 \mid \mathrm{C}_{\mathrm{n}-1}}=\mathrm{j}^{\mathrm{k}} \mathrm{p}_{\mathrm{n}} \mathrm{C}_{\mathrm{n}} \circ \mathrm{j}^{\mathrm{k}} \varphi_{\mathrm{n}, \mathrm{n}-1 \mid \mathrm{C}_{\mathrm{n}-1}}, \\
& \mathrm{j}^{\mathrm{k}} \mathrm{p}_{\mathrm{n}_{\mid \mathrm{C}_{\mathrm{n}}}}=\mathrm{j}^{\mathrm{k}} \mathrm{p}_{1 \mid \mathrm{C}_{1}} \circ \mathrm{j}^{\mathrm{k}} \varphi_{1, \mathrm{n} \mid \mathrm{C}_{\mathrm{n}}},
\end{aligned}
$$

where $\phi_{i+1, i}, i=1, . ., n(\bmod n)$ are the connecting diffeomorphisms.

Substituting subsequently gives

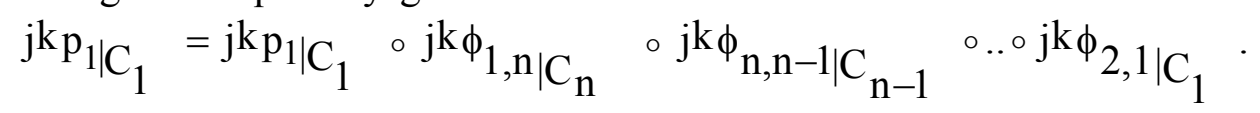

Since the jet $\mathrm{jkp}_{1 \mid \mathrm{C}_{1}}$ is left-invertible, the following necessary condition follows:

7.1 Theorem

The connecting diffeomorphisms $\phi_{i+1, i}$ between subsequent patches $p_{i}$.(i=1,..,n $(\bmod n))$ surrounding a vertex $\mathrm{Q}=\mathrm{p}_{\mathbf{i}}\left(\mathrm{C}_{\mathrm{i}}\right)$ with $\mathrm{GC}{ }^{\mathrm{k}}$ satisfy:

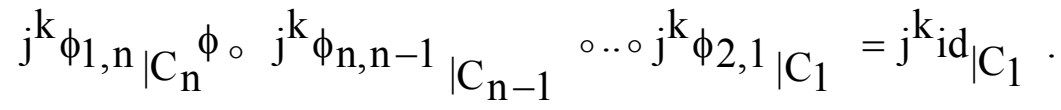

7.2 Example: Assume three patches are defined on the unit square or the triangle with vertices $0=(0,0),(1,0),(0,1)$ and surround a vertex with $\mathrm{GC}^{2}$, with connecting diffeomorphisms $\phi, X, \psi$, from edge $(0, s)$ to $(s, 0)$. Then the necessary condition reads:

$$
\begin{aligned}
& \psi(X(\phi(0)))=0 \text {, } \\
& \left.\partial \psi\right|_{0} \circ \partial X_{\mid 0} \circ \partial_{\phi \mid 0}=\mathrm{id},
\end{aligned}
$$

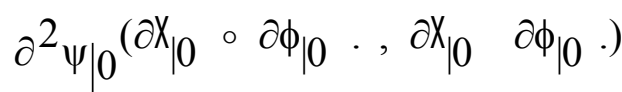

$$
\begin{aligned}
& +\partial^{2} \psi \mid 0 \quad \circ \partial^{2} \chi_{\mid 0}(\partial \phi|0 \quad . \partial \phi| 0 \quad .) \\
& +\left.\left.\partial \psi\right|_{0} \circ \partial^{2} \chi_{\mid 0} \partial^{2} \phi\right|_{0} \quad(., .) \equiv 0 \text {. }
\end{aligned}
$$


Writing in terms of shape parameters $\lambda_{i}, \mu_{i}, \omega_{i}, \rho_{i}, \sigma_{i}, \tau_{i}(i=1,2,3)$, where $\lambda_{1}:=\mathrm{v}_{1}(0), \mu_{1}:=\mathrm{v}_{2}(0), \omega_{1}:=\mathrm{v}_{1}^{\prime}(0), \rho_{1}:=\mathrm{v}_{2}^{\prime}(0), \sigma_{1}:=\mathrm{w}_{1}(0), \tau_{1}:=\mathrm{w}_{2}(0)$ describe $\phi$ according to example 3.4 , and similarly $\lambda_{2}, \ldots, \tau_{2}$ and $\lambda_{3}, \ldots, \tau_{3}$ describe $\mathrm{X}$ and $\psi$ respectively, gives, after some calculation:

$$
\begin{aligned}
& \mu_{1} \mu_{2} \mu_{3}=-1, \\
& \lambda_{\mathrm{i}} \mu_{\mathrm{i}+1}=1, \text { for } \mathrm{i}=1,2,3(\bmod 3) \\
& \lambda_{1}^{2} \lambda_{3} \sigma_{2}+\lambda_{1}^{2} \tau_{2}+2 \lambda_{1} \omega_{2}+2 \lambda_{1} \mu_{1} \rho_{2}+\lambda_{2} \tau_{1}=0, \\
& \lambda_{1}^{2} \mu_{3} \sigma_{2}-2 \lambda_{1}^{2} \omega_{2}+\sigma_{1}+\mu_{3} \tau_{1}=0, \\
& \lambda_{2} \omega_{3}+\lambda_{1} \lambda_{3} \sigma_{2}+\lambda_{1} \tau_{2}+\omega_{2}+\mu_{1} \rho_{2}+\lambda_{3} \rho_{1}=0 \\
& \lambda_{2} \rho_{3}+\lambda_{1} \mu_{3} \sigma_{2}+\mu_{1} \mu_{3} \omega_{2}+\omega_{1}+\mu_{3} \rho_{1}=0 \\
& \lambda_{2}^{2} \sigma_{3}+2 \mu_{2} \lambda_{2} \omega_{3}+\lambda_{3} \sigma_{2}+\tau_{2}=0 \\
& \lambda_{2}^{2} \tau_{3}+2 \mu_{2} \lambda_{2} \rho_{3}+\mu_{3} \sigma_{2}=0 .
\end{aligned}
$$

The first two equations are the $\mathrm{GC}^{1}$-conditions and accord with those of [Höllig '86]. Note that the $\mathrm{GC}^{2}$-conditions are easily satisfied if $\omega_{i}=\rho_{i}=\sigma_{i}=\tau_{i}=0$, i.e. the necessary condition for higher order geometric continuity is satisfied automatically, if the $\mathrm{GC}^{1}$-condition is met and the higher derivatives of the connecting diffeomorphisms vanish at the corner points.

In the applications (Sec. 8 and 9) connecting diffeomorphisms will be constructed that satisfy the condition of theorem 7.1. Then patches are defined appropriately such that abutting patches join $\mathrm{GC}$ with these connecting diffeomorphisms. From the explicit construction of these patches it will be clear that $\mathrm{GC}^{\mathrm{k}}$ is also achieved at the vertices.

However, $\mathrm{GC}^{\mathrm{k}}$ at the vertices is guaranteed more generally by the following theorem. Since it is mainly of theoretical interest, the proof is sketched only.

\subsection{Theorem:}

Let $\Delta \subset \mathbb{R}^{2}$ be domains with corners $\mathrm{C}_{\mathbf{i}}$ of adjoining edges $\mathrm{E}_{\mathrm{i}+}(\mathrm{s}), \mathrm{E}_{\mathrm{i}-}(\mathrm{s})$, $\mathrm{s} \in[0,1], \mathrm{C}_{\mathrm{i}}=\mathrm{E}_{\mathrm{i}+}(0)=\mathrm{E}_{\mathrm{i}-}(0)$, and let $\phi_{\mathrm{i}+1}, \mathrm{i}$ be $\mathrm{C}^{\mathrm{k}}$-connecting diffeomorphisms from $\mathrm{E}_{\mathrm{i}+}$ to $\mathrm{E}_{\mathrm{i}+1-}, \mathrm{i}=1, . ., \mathrm{n}(\bmod \mathrm{n})$. 
Assume that the jets at the corners satisfy

$$
\mathrm{j}^{\mathrm{k}} \varphi_{1, \mathrm{n}} \mathrm{C}_{\mathrm{n}} \circ \mathrm{j}^{\mathrm{k}} \varphi_{\mathrm{n}, \mathrm{n}-1 \mid \mathrm{C}_{\mathrm{n}-1}} \circ ., \circ \mathrm{j}^{\mathrm{k}} \varphi_{2,1 \mid \mathrm{C}_{1}}=\mathrm{j}^{\mathrm{k}} \mathrm{id}_{\mid \mathrm{C}_{1}}
$$

and that the tangent sectors (in $\mathbb{R}^{2}$ ) of

$$
\begin{gathered}
\Delta_{\mathrm{n}} \text { at } \mathrm{C}_{\mathrm{n}}, \\
\phi_{\mathrm{n}, \mathrm{n}-1_{1}} \text { at } \mathrm{C}_{\mathrm{n}_{-1}}, \\
\phi_{\mathrm{n}, \mathrm{n}-1} \circ \phi_{\mathrm{n}_{-1, \mathrm{n}_{-2}}} \text { at } \mathrm{C}_{\mathrm{n}_{-2},} \\
\cdot \cdot \cdot \\
\phi_{{\mathrm{n}, \mathrm{n}_{-1}}} \circ \phi_{\mathrm{n}_{-1, \mathrm{n}_{-2}}} \circ \cdot \phi_{2,1} \text { at } \mathrm{C}_{1}
\end{gathered}
$$

do not overlap, see fig . 3 .

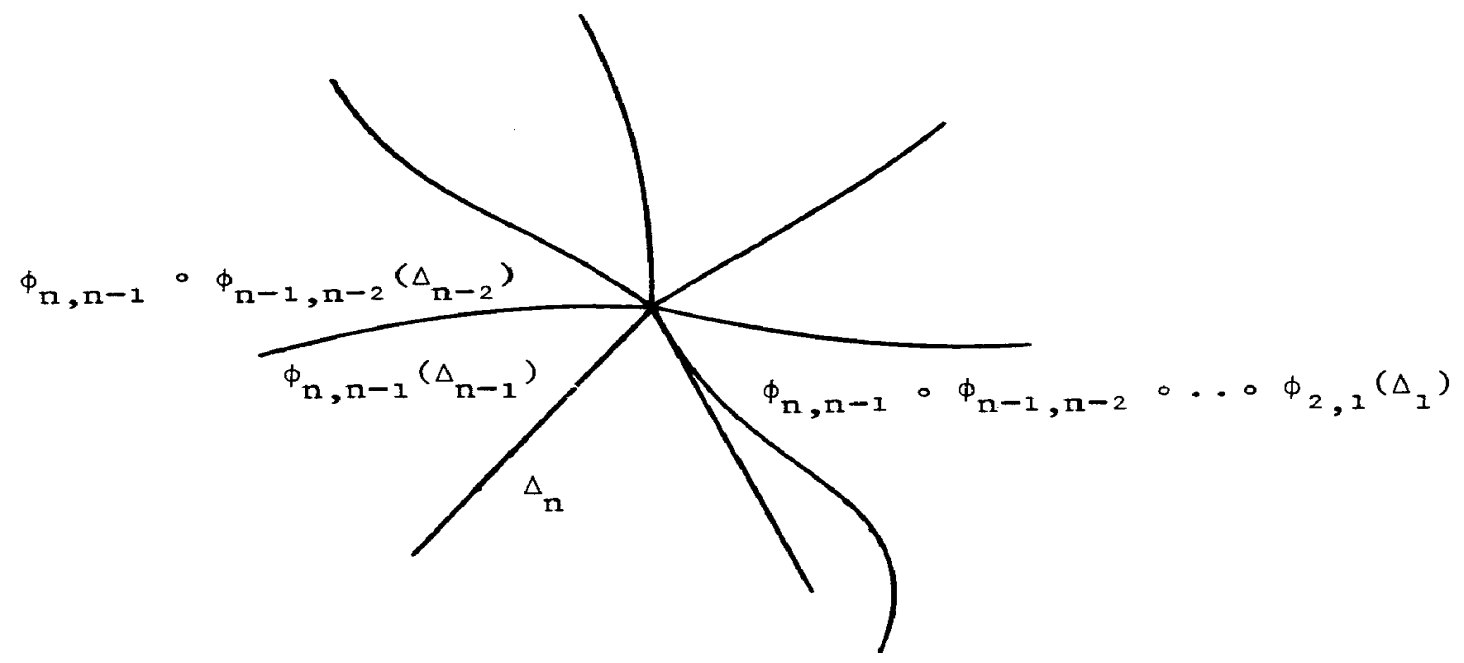

Fig. 3

Then :

The disjoint union of the domains $\Delta_{i}$, modulo boundary identifications, can be given a $\mathrm{C}^{\mathrm{k}}$-differentiable structure such that a function on this space is $\mathrm{C}^{\mathrm{k}}$ iff its restrictions to $\Delta_{\mathrm{i}}$ are $\mathrm{C}^{\mathrm{k}}$ and join $\mathrm{GC}^{\mathrm{k}}$.

More precisely:

Let $\Delta^{\circ}:=\bigcup_{i=1, . ., n} \Delta_{i}$ be the disjoint union of the domains. The projection of $\Delta^{\circ}$ onto the quotient space $\Delta$, obtained by identifying $\mathrm{E}_{\mathrm{i}+}(\mathrm{s})$ with $\mathrm{E}_{\mathrm{i}+\mathrm{i}-}(\mathrm{s})$, gives rise to embeddings $\pi_{i}: \Delta_{i} \rightarrow \Delta$. 
Then A admits a $\mathrm{C}^{\mathrm{k}}$-differentiable structure such that a function $\mathrm{p}: \Delta \rightarrow \mathrm{IR}^{3}$ is $\mathrm{C}^{\mathrm{k}}$ iff the functions $\mathrm{p}_{\mathrm{i}}:=\mathrm{p} \circ \pi_{\mathrm{i}}: \Delta_{\mathrm{i}} \rightarrow \mathbb{R}^{3}$ are $\mathrm{C}^{\mathrm{k}}$ and $\mathrm{p}_{\mathrm{i}}, \mathrm{p}_{\mathrm{i}+1}$ join $\mathrm{GC}^{\mathrm{k}}$ with connecting diffeomorphisms $\phi_{i_{+1}, i}$

Proof: The crucial step is to construct a coordinate chart around the vertex $\mathrm{C}:=\pi_{\mathrm{i}}\left(\mathrm{C}_{\mathrm{i}}\right)$.

Choose a neighbourhood $\Omega$ of $\mathrm{C}$ in $\Delta$ such that

$$
\phi_{\mathrm{n}, \mathrm{n}_{-1}} \circ \cdots \circ \phi_{\mathrm{i}_{+1, \mathrm{i}}} \text { is defined on } \pi_{\mathrm{i}}^{-1}(\Omega) \text { for } \mathrm{i}=1, \ldots, \mathrm{n}-1 \text {, }
$$

and $\phi_{\mathrm{n}, 1}\left(:=\phi_{1, \mathrm{n}}^{-1}\right)$ is defined on $\pi_{\mathrm{i}}^{-1}(\Omega)$.

The vertex condition implies that

$$
\mathrm{j}^{\mathrm{k}} \phi_{\mathrm{n}, 1 \mid \mathrm{C}_{1}}=\mathrm{j}^{\mathrm{k}}\left(\phi_{\mathrm{n}, \mathrm{n}-1} \circ \cdots \circ \phi_{21}\right)_{\mid} \mathrm{C}_{1},
$$

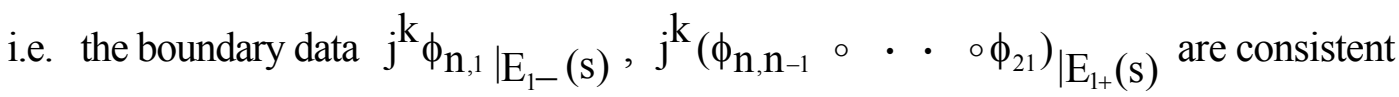
and there exists a $\mathrm{C}^{\mathrm{k}}$-diffeomorphism $\phi$, defined on an open set containing $\pi_{i}^{-1}(\Omega)$, that matches these boundary data. The sectors $\phi\left(\pi_{1}^{-1}(\Omega)\right), \phi_{n, n-1} \circ \cdots \circ \phi_{i+1, i}\left(\pi_{i}^{-1}(\Omega)\right)$ $(\mathrm{i}=2, . ., \mathrm{n}-1)$ and $\pi_{\mathrm{n}}^{-1}(\Omega)$ cover a neighbourhood of $\mathrm{C}_{\mathrm{n}} \quad$ (in $\mathbb{R}^{2}$ and have disjoint interiors, due to the non-overlap assumption. The map $\psi_{\mathrm{C}}$ defined by

$$
\psi_{C}(X):= \begin{cases}\pi_{n}^{-1}(X), & \text { for } X \in \pi_{n}\left(\Delta_{n}\right), \\ \phi_{n, n-1} \circ & \circ \phi_{i+1, i}\left(\pi_{i}^{-1}(X)\right), \quad \text { for } X \in \pi_{i}\left(\Delta_{i}\right)(i=2, . ., n-1), \\ \phi\left(\pi_{1}^{-1}(X)\right), & \text { for } X \in \pi_{1}\left(\Delta_{1}\right)\end{cases}
$$

maps $\Omega$ homeomorphically onto this neighbourhood, i.e. $\psi_{\mathrm{C}}$ is a coordinate chart around the vertex.

For an edge point $\pi_{i}\left(E_{i+}(s)\right), s \neq 0$, a coordinate chart $\psi_{i}$ is given by

$$
\psi_{i}(X):= \begin{cases}\pi_{i+1}^{-1}(X), & \text { if } \quad X \in \pi_{i+1}\left(\Delta_{i+1}\right), \\ \phi_{i+1, i}\left(\pi_{i}^{-1}(X)\right) & \text { if } X \in \pi_{i}\left(\Delta_{i}\right),\end{cases}
$$

and for $X \in \pi_{i}\left(\Delta_{i}\right), \pi_{i}^{-1}(X)$ not on an edge, $\pi_{i}^{-1}$ is a coordinate chart. All these charts have $\mathrm{C}^{\mathrm{k}}$ coordinate changes.

The conclusion for functions on $\Delta$ follows since a function $\mathrm{p}$ on $\Delta$ is $\mathrm{C}^{\mathrm{k}}$ iff its local representations $\mathrm{p} \circ \psi^{-1}$ are $\mathrm{C}^{\mathrm{k}}$ for all coordinate charts $\psi=\psi_{\mathrm{C}}, \psi_{\mathrm{i}}, \pi_{\mathrm{i}}^{-1}$. 
Note that if patches $\mathrm{p}_{\mathrm{i}}$ surround a vertex with $\mathrm{GC}^{\mathrm{k}}$, then $\mathrm{p} \circ \psi_{\mathrm{C}}^{-1}$ is a $\mathrm{C}^{\mathrm{k}}$-reparameterization around the vertex.

This theorem gives the connection to [DeRose '85]. The manifold $\Delta$ can be given even a $\mathrm{C}^{\infty}$-structure, of. [Hirsch '76], This is the domain manifold on which DeRose's 'abstract spline' is defined. The difference is, that for his theory this manifold must be known, thus moving the problem of geometric continuity to that of finding an abstract manifold, while here geometric continuity is treated only in terms of patches and connecting diffeomorphisms and the manifold $\Delta$ need not be considered.

\section{Geometric Continuous Patch Complexes for Modelling Closed Surfaces}

Now the theory is applied to the problem of closed surfaces.

8.1 Definition: A closed $\mathrm{C}^{\mathrm{k}} \underline{\text { Surface }}$ is a $\mathrm{GC}^{\mathrm{k}}$-patch complex whose connecting relation links each edge with exactly one other edge.

\subsection{Generating a closed surface; Consider a closed surface defined by a} network of control-points with 4-sided meshes where an arbitrary number of meshes is allowed to meet at a control-point. This network is approximated by a $\mathrm{GC}^{\mathrm{k}}$-patch complex consisting of rectangular patches, each patch corresponding to a 4-sided mesh, and the connecting relation linking edges that correspond to coinciding sides of the respective meshes. The details of this construction are given in [Gregory and Hahn '87b]

The major step is to determine the connecting diffeomorphisms:

The necessary condition (theorem 7.1) for the connecting diffeomorphisms of $n$ rectangular patches surrounding a vertex with $\mathrm{GC}^{\mathrm{k}}$, means for the first derivative, written in terms of shape parameters $\lambda_{i}, \mu_{i}$, as in example 7.2 :

$$
\left(\begin{array}{ll}
\lambda_{n} & 1 \\
\mu_{n} & 0
\end{array}\right)\left(\begin{array}{ll}
\lambda_{n-1} & 1 \\
\mu_{n-1} & 0
\end{array}\right) \cdots\left(\begin{array}{ll}
\lambda_{1} & 1 \\
\mu_{1} & 0
\end{array}\right)=\left(\begin{array}{ll}
1 & 0 \\
0 & 1
\end{array}\right)
$$


A symmetric solution of this equation is

$$
\lambda_{i}=2 \cos \frac{2 \pi}{n}, \mu_{i}=-1,
$$

and the condition for the whole k-jets is met, if the higher order derivatives vanish at $(0,0)$.

Diffeomorphisms with jets of this kind at the corners can be taken as connecting diffeomorphisms. To fill in abutting meshes by patches $\mathrm{p}, \mathrm{q}$ where the common side of the meshes corresponds to say edge $(0, s)$ of $p$ and edge $(\mathrm{s}, 0)$ of $\mathrm{q}$, and where at the vertices (corresponding to $\mathrm{s}=0, \mathrm{~s}=1) \mathrm{n}_{0}$ and $\mathrm{n}_{1}$ meshes meet respectively, the connecting diffeomorphism can be set as

$$
\phi(r, s):=\left(s+2 r\left(\alpha(s) \cos \frac{2 \pi}{n_{0}}-\beta(s) \cos \frac{2 \pi}{n_{1}},-r\right),\right.
$$

where $\alpha$ and $\beta$ are $\mathrm{C}^{\mathrm{k}}$-functions such that

$$
\begin{aligned}
& \alpha(s), \beta(s) \geq 0, \\
& \alpha(0)=\beta(1)=1, \alpha(1)=\beta(0)=0
\end{aligned}
$$

and all derivatives up to order $\mathrm{k}$ at 0 and 1 vanish.

If, for each side of the network, a connecting diffeomorphism of this form is chosen, then the meshes can be filled in by patches that join $\mathrm{GC}^{\mathrm{k}}$, with these connecting diffeomorphisms, Moreover, all vertices are surrounded with $\mathrm{GC}^{\mathrm{k}}$, i.e. the patches form a $\mathrm{GC}^{\mathrm{k}}$ patch complex.

9. Filling a Triangular Patch into a Geometric Continuous Patch Complex

9.1 The problem: Assume that patches form a $\mathrm{GC}^{\mathrm{k}}$-patch complex around a triangular hole with vertices $Q_{i}$ see fig.4, such that:

(i) patches $\mathrm{p}_{\mathrm{i}}, \mathrm{i}=1,2,3(\bmod 3)$ abut onto the triangular hole with adjoining edges $e_{i}(s), s \in[0,1]$ where

(ii) $\mathrm{p}_{\mathrm{i}_{-1}}, \mathrm{p}_{\mathrm{i}}$, and possibly more patches, meet $\mathrm{GC}^{\mathrm{k}}$ with a non-convex corner at $\mathrm{Q}_{\mathrm{i}}$. 


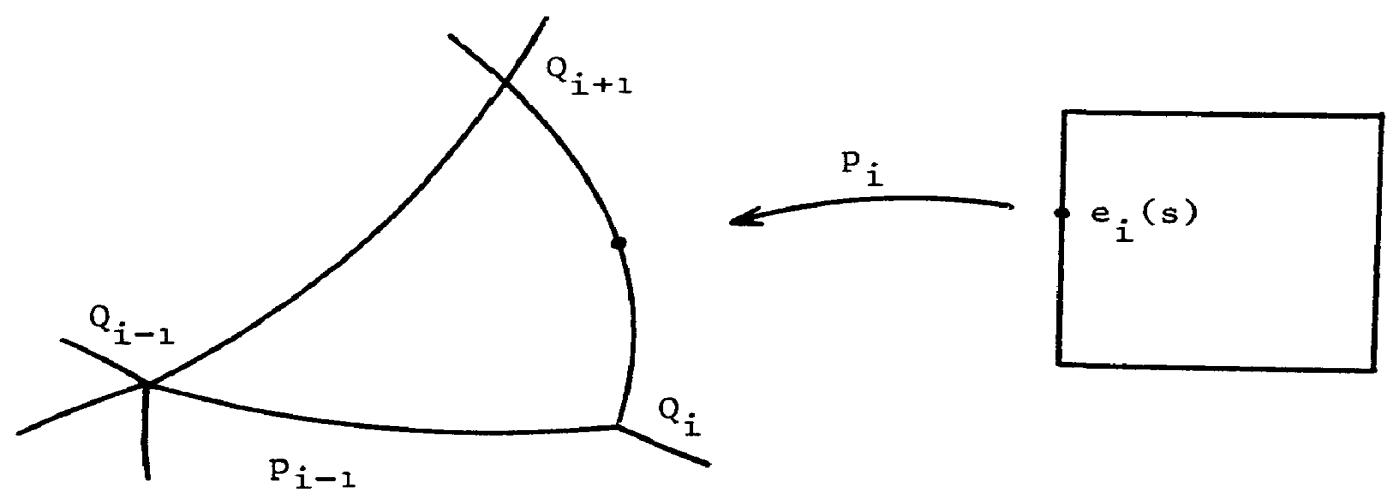

Fig. 4

The hole is to be filled in by a patch $\mathrm{P}$, defined on an equilateral triangle with vertices $C_{i}$ and edges $E_{i}(s)=(1-s) C_{i}+s_{i+1}$.

9.2 Outline of the construction: In order to have $\mathrm{GC}^{\mathrm{k}}$-joins with the abutting patches $\mathrm{p}_{i}$, the patch $\mathrm{P}$ must satisfy the conditions

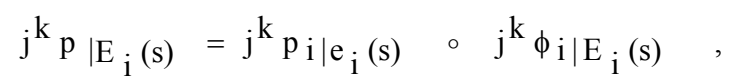

with connecting diffeomorphisms $\phi_{i}$ from $E_{i}(s)$ to $e_{i}(s)$.

Necessary for the existence of such a patch is that its jets are well-defined at the corners, i.e.

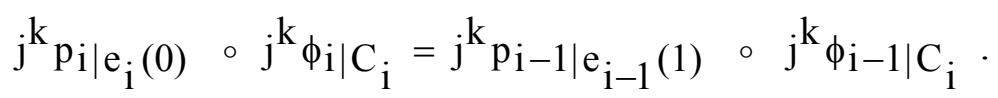

This is equivalent to the condition of theorem 7.1 for the patches surrounding the vertex $Q_{i}$, which here says that

$$
\mathrm{j}^{\mathrm{k}} \phi_{\mathrm{i}-1 \mid \mathrm{C}_{\mathrm{i}}}=\mathrm{j}^{\mathrm{k}} \phi_{\mathrm{i}-1, \mathrm{i} \mid \mathrm{e}_{\mathrm{i}}(0)} \circ \mathrm{j}^{\mathrm{k}} \phi_{\mathrm{i} \mid \mathrm{C}_{\mathrm{i}}} .
$$

where $j^{k} \phi_{i-1, i \mid e_{i}(0)}$ is the jet composed of the jets of the connecting diffeomorphisms between subsequent patches $p_{i}, . ., p_{i-1}$ meeting at $Q_{i}$.

Now the triangular patch $\mathrm{P}$ can be obtained as follows:

Construct $\mathrm{C}^{\mathrm{k}}$-connecting diffeomorphisms $\phi_{\mathrm{i}}$ satisfying (2). Then the boundary data (1) are consistent with a $\mathrm{C}^{\mathrm{k}}$-function on the triangle. Any interpolant to these boundary data joins $\mathrm{GC}^{\mathrm{k}}$ along the edges and also at the corners. The details of this construction are given in [Hahn '87]. 
9.3 Example: In the special case where the triangular hole is surrounded by rectangular patches meeting with $\mathrm{C}^{2,2}$-parametric continuity, a triangular patch with $\mathrm{GC}^{2}$-joins is explicitly:

$$
P(X)=\sum_{i=1}^{3} \frac{b_{i}^{3}}{\sum_{j=1}^{3} b_{j}^{3}} P_{i}\left(b_{i+1}, b_{i+2}\right)
$$

where $\left(b_{1}, b_{2}, b_{3}\right)$ are the barycentric coordinates of $X$ with respect to $\mathrm{C}_{1}, \mathrm{C}_{2}, \mathrm{C}_{3}$ and $\mathrm{P}_{\mathrm{i}}(\mathrm{s}, \mathrm{t})=\mathrm{p}_{\mathrm{i}}(0, \mathrm{~s})$

$$
\begin{aligned}
& +t\left(-\partial_{10} p_{i}(0, s)+\beta(s) \partial_{01} p_{i}(0, s)\right) \\
& +\frac{t^{2}}{2}\left(\partial_{20} p_{i}(0, s)-2 \beta(s) \partial_{11} p_{i}(0, s)\right. \\
& \quad+\beta^{2}(s) \partial_{02} p_{i}(0, s) \\
& \quad+\beta^{\prime}(s) \partial_{01} p_{i}(0, s) \\
& +p_{i-1}(0,1-t) \\
& +s\left(-\partial_{10} p_{i_{-1}}(0,1-t)-\alpha(1-t) \partial_{01} p_{i-1}(0,1-t)\right) \\
& +\frac{s^{2}}{2}\left(\partial_{20} p_{i-1}(0,1-t)+2 \alpha(1-t) \partial_{11} p_{i_{-1}}(0,1-t)\right. \\
& \quad+\alpha^{2}(1-t) \partial_{02} p_{i-1}(0,1-t) \\
& \quad+\alpha^{\prime}(1-t) \partial_{01} p_{i_{-1}}(0,1-t) \\
& -p_{i}(0,0) \\
& -s_{01} p_{i}(0,0)+t \partial_{10} p_{i}(0,0) \\
& -\frac{s^{2}}{2} \partial_{02} p_{i}(0,0)+s t \partial_{11} p_{i}(0,0)-\frac{t^{2}}{2} \partial_{20} p_{i}(0,0) \\
& +\frac{s^{2} t^{2}}{2} \partial_{12} p_{i}(0,0)-\frac{s t^{2}}{2} \partial_{21} p_{i}(0,0) \\
& -\frac{s^{2} t^{2}}{4} \partial_{22} p_{i}(0,0)
\end{aligned}
$$

Here $\alpha, \beta$ are $C^{3}$-functions as in paragraph 8.2 To guarantee second order differentiability of the patch $\mathrm{P}$, it must be assumed that the abutting patches are $\mathrm{C}^{4}$. 
Appendix; Differential Geometric Invariants

First and second order geometric continuity of curves and surfaces have been described by many authors using geometric invariants (tangents, curvatures). A parameterisation independent characterization of higher order geometric continuity is provided by the covariant derivative from differential geometry, of [DoCarmo '76], [Spivak '79], [Kobayashi et al. '63].

A. 1 Definition: Let $\mathrm{p}: \Delta \rightarrow \mathbb{R}^{3}$ be a $\mathrm{C}^{\mathrm{k}}$-patch with unit normal vector field $\mathrm{N}: \Delta \rightarrow \mathbb{R}^{3}$.

If $X(t)$ is a curve in $A$ and $W(t)\left(\right.$ in $\left.\mathbb{R}^{3}\right)$ is a tangent vector field along $X(t)$, i.e. there is a vector field $U(t)\left(\right.$ in $\mathbb{R}^{3}$ ) such that $W(t)=\partial p_{\mid X(t)} U(t)$, then the covariant derivative of $\mathrm{W}$ is the tangent component of the ordinary (euclidean) derivative:

$$
\frac{\mathrm{D}}{\mathrm{dt}} \mathrm{W}(\mathrm{t}):=\frac{\mathrm{d}}{\mathrm{dt}} \mathrm{W}(\mathrm{t}), \mathrm{N}(\mathrm{X}(\mathrm{t}))>\mathrm{N}(\mathrm{X}(\mathrm{t}))
$$

Here $<\bullet, \bullet>$ denotes the euclidean scalar product in $\mathbb{R}^{3}$.

The covariant differential of order $\mathrm{j}(\mathrm{j} \leq \mathrm{k})$ of the normal field at a point $\mathrm{X} \in \Delta$ acts on a $\mathrm{j}$-tuple of tangent vectors $\mathrm{W}_{1}, \ldots, \mathrm{W}_{\mathrm{j}}\left(\right.$ in $\mathbb{R}^{3}$ ) and is defined recursively by

$$
\begin{aligned}
& \mathrm{DN}_{\mid X}\left(\mathrm{~W}_{1}\right):=\frac{\mathrm{D}}{\mathrm{dt}} \mathrm{N}(\mathrm{X}(\mathrm{t}))_{\mid \mathrm{t}=0}:=\frac{\mathrm{d}}{\mathrm{dt}} \mathrm{N}(\mathrm{X}(\mathrm{t}))_{\mid \mathrm{t}=0} \\
& D^{\mathrm{j}_{N}}{ }_{\mid X}\left(\mathrm{~W}_{1}, . ., \mathrm{W}_{\mathrm{j}}\right):=\frac{\mathrm{D}}{\mathrm{dt}} \mathrm{D}^{\mathrm{j}-1} \mathrm{~N}_{\mid X(\mathrm{t}))}\left(\mathrm{W}_{2}(\mathrm{t}), . ., \mathrm{W}_{\mathrm{j}}(\mathrm{t})\right)_{\mid \mathrm{t}=0} \\
& -\sum_{i=2}^{j} D^{j-1} N_{\mid X}\left(W_{2}, . ., \frac{D}{d t} W_{i}(0), . ., W_{j}\right),
\end{aligned}
$$

where $\mathrm{X}(\mathrm{t})$ is a curve in $\Delta$ with $\mathrm{X}(0)=\mathrm{X}$ and $\mathrm{W}_{1}=\left(\begin{array}{lll}\mathrm{p} & \circ \mathrm{X}\end{array}\right)^{\cdot}(0)$, and $\mathrm{W}_{\mathrm{i}}(\mathrm{t}), \mathrm{i}=2, . ., \mathrm{j}$ are tangent vector fields along $X(t)$ with $W_{i}(0)=W_{i}$.

The following theorem develops an idea of [DeRose '85].

A, 2 Theorem;

Assume $\mathrm{C}^{\mathrm{k}}$-patches $\mathrm{p}_{1}, \mathrm{p}_{2}$ abut with a common boundary curve

$$
\mathrm{p}_{1}\left(\mathrm{E}_{1}(\mathrm{~s})\right)=\mathrm{p}_{2}\left(\mathrm{E}_{2}(\mathrm{~s}), \mathrm{s} \in[0,1]\right. \text {. }
$$


Then $\mathrm{p}_{1}, \mathrm{p}_{2}$ join $\mathrm{GC}^{\mathrm{k}}$ along $\mathrm{E}_{1}, \mathrm{E}_{2}$ iff the common boundary curve is not a ridge (i.e. the tangent sectors of $\mathrm{p}_{1}$ at $\mathrm{E}_{1}(\mathrm{~s})$ and of $\mathrm{p}_{2}$ at $\mathrm{E}_{2}(\mathrm{~s})$ do not overlap) and there exist normal vector fields $\mathrm{N}_{1}, \mathrm{~N}_{2}$ of $\mathrm{p}_{1}, \mathrm{p}_{2}$ respectively such that

$$
\mathrm{D}^{\mathrm{j}} \mathrm{N}_{1 \mid \mathrm{E}_{1}(\mathrm{~s})}=\mathrm{D}^{\mathrm{j}} \mathrm{N}_{2 \mid \mathrm{E}_{2}(\mathrm{~s})}, \quad 0 \leq \mathrm{j} \leq \mathrm{k}-1, \quad \mathrm{~s} \in[0,1] \text {. }
$$

Proof: The condition on the covariant differential is necessary for $\mathrm{GC}^{\mathrm{k}}$, since it is independent of parameterization.

To prove sufficiency, let $\mathrm{W}(\mathrm{s})$ be a tangent vector field along $\mathrm{E}_{1}(\mathrm{~s})$ transversal to $\mathrm{p}_{1} \circ \mathrm{E}_{1}(\mathrm{~s})$ and pointing towards patch $\mathrm{p}_{1}$. Choose coordinate transformations $\xi_{1}(\mathrm{~s}, \mathrm{t}), \xi_{2}(\mathrm{~s}, \mathrm{t})$ such that

$$
\begin{aligned}
& \xi_{1}(\mathrm{~s}, 0)=\mathrm{E}_{1}(\mathrm{~s}), \xi_{2}(\mathrm{~s}, 0)=\mathrm{E}_{2}(\mathrm{~s}, 0) \\
& \frac{\partial}{\partial \mathrm{t}}\left(\mathrm{p}_{1} \circ \xi_{1}\right)(\mathrm{s}, 0)=\mathrm{W}(\mathrm{s})=\frac{\partial}{\partial \mathrm{t}}\left(\mathrm{p}_{2} \circ \xi_{2}\right)(\mathrm{s}, 0)
\end{aligned}
$$

and

$$
\frac{\partial^{j-2}}{\partial t^{j-2}} \frac{D}{d t} \frac{\partial}{\partial t}\left(p_{i} \circ \xi_{i}\right)(s, 0)=0 \text { for } j=2, . ., k, i=1,2 .
$$

Note that $\mathrm{p}_{1} \circ \xi_{1}(\mathrm{~s}, \mathrm{t})$ is defined for $\mathrm{t} \geq 0$ and $\mathrm{p}_{2} \circ \xi_{2}(\mathrm{~s}, \mathrm{t})$ for $\mathrm{t} \leq 0$. The crossboundary derivatives of $\mathrm{p}_{1} \circ \xi_{1}(\mathrm{~s}, \mathrm{t})$ at $(\mathrm{s}, 0)$ are

$$
\begin{gathered}
\frac{\partial}{\partial \mathrm{t}}\left(\mathrm{p}_{\mathrm{i}} \circ \xi_{\mathrm{i}}\right)(\mathrm{s}, 0)=\mathrm{W}(\mathrm{s}), \\
\frac{\partial^{2}}{\partial \mathrm{t}^{2}}\left(\mathrm{p}_{\mathrm{i}} \circ \xi_{\mathrm{i}}\right)(\mathrm{s}, 0)=-<\mathrm{W}(\mathrm{s}), \frac{\partial}{\partial \mathrm{t}}\left(\mathrm{N}_{\mathrm{i}} \circ \xi_{\mathrm{i}}\right)(\mathrm{s}, 0)>\mathrm{N}_{\mathrm{i}} \circ \xi_{\mathrm{i}}(\mathrm{s}, 0),
\end{gathered}
$$

and - by induction - the higher derivatives can be written in terms of derivatives of $\frac{\mathrm{D}}{\mathrm{dt}} \frac{\partial}{\partial \mathrm{t}} \mathrm{p}_{\mathrm{i}} \circ \xi_{\mathrm{i}} \quad$ (which vanish) and $\frac{\partial^{\mathrm{j}}}{\partial \mathrm{t}} \mathrm{N}_{\mathrm{i}} \circ \xi_{\mathrm{i}}$ which can be entirely expressed by covariant derivatives of $\mathrm{N}_{\mathrm{i}} \circ \xi_{\mathrm{i}}$. Since these are

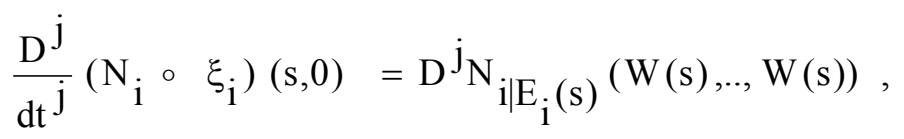

the derivatives of $\mathrm{p}_{1} \circ \xi_{1}$ and $\mathrm{p}_{2} \circ \xi_{2}$ coincide up to order $\mathrm{k}$ along $(\mathrm{s}, 0)$, i,e, $\mathrm{p}_{1} \mathrm{p}_{2}$ join $\mathrm{GC}^{\mathrm{k}}$ with connecting diffeomorphism $\xi_{2} \circ \xi_{1}^{-1}$.

In case $\mathrm{k}=1$, the condition of the theorem is known as tangent plane continuity. For $\mathrm{k}=2$ the covariant differential $\mathrm{DN}$ is the shape operator (up to sign). Several authors have based their definition of $\mathrm{GC}^{2}$ on the shape operator [Jensen '85] 
or equivalents as the principal curvatures and directions [Vernon et al '76],

[Herron '86], or the Dupin indicatrix [Kahmann '83].

However, the theorem is not used in the construction $\mathrm{GC}^{\mathrm{k}}$ patches. Rather it provides a test for $\mathrm{GC}^{\mathrm{k}}$, since the covariant derivatives in a transversal direction can be computed straightforwardly.

\section{$\underline{\text { References }}$}

Barnhill, R.E. (1985), Surfaces in computer aided geometric design: A survey with new results, Computer Aided Geometric Design 2, 1-17.

Barsky, B.A. and DeRose, T.D. (1985), An intuitive approach to geometric continuity for parametric curves and surfaces, in N. Magenay and D. Thalmann, eds, Computer Generated Images - The State of the Art, Springer, pp. 159-175.

DeRose, T.D. (1985), Geometric continuity: a parametrization independent measure of continuity for computer aided geometric design, $\mathrm{Ph} . \mathrm{D}$. thesis, Computer Science Division, University of California at Berkeley.

DoCarmo, M.P. (1976), Differential Geometry of Curves and Surfaces, Prentice Hall, New Jersey.

Gregory, J.A. and Hahn, J.M. (1986), Geometric continuity and convex combination patches, to appear in Computer Aided Geometric Design.

Gregory, J.A. and Hahn, J.M. (1987a), A C ${ }^{2}$ - polygonal surface patch, Mathematics Department TR/06/87, Brunel University, in these proceedings.

Gregory, J.A. and Hahn, J.M. (1987b), Geometric continuous patch complexes for modelling closed surfaces, in preparation.

Hahn, J.M. (1987), Triangular patches within a geometric continuous patch complex, Mathematics Department TR/07/87, Brunel University.

Hirsch, M.W. (1976), Differential Topology, Springer Graduate Texts in Mathematics 33.

Höllig, K. (1986), Geometric continuity of spline curves and surfaces, Computer Sciences Technical Report 645, University of Wisconsin, Madison.

Jensen, T. (1985), Assembling triangular and rectangular patches and multivariate splines, Evans \& Sutherland Computer Corp.

Kahmann, J. (1983), Continuity of curvature between adjacent Bézier patches, in R.E. Barnhill and W, Böhm, eds., Surfaces in Computer Aided Geometric Design, North-Holland, Amsterdam, pp. 65-75.

Kobayashi, S. and Nomizu, K. (1963), Foundations of Differential Geometry, vol. I , Interscience, NY.

Spivak, M. (1979), A Comprehensive Introduction to Differential Geometry, vol. II , Publish or Perish, Berkeley.

Veron, M. Ris, G. and Musse, J.P. (1976), Continuity of biparametric surface patches, Computer Aided Design 8, 267-273. 


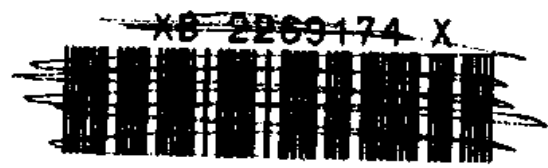

